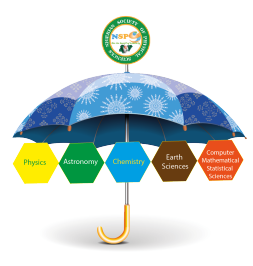

\title{
Stability and Sensitivity Analysis of Dengue-Malaria Co-Infection Model in Endemic Stage
}

\author{
Solomon Akyenyi Ayuba ${ }^{\mathrm{a}, *}$, Imam Akeyede ${ }^{\mathrm{a}}$, Adeyemi Sunday Olagunju ${ }^{\mathrm{a}, \mathrm{b}}$ \\ ${ }^{a}$ Department of Mathematics Federal University of Lafia, Nigeria \\ ${ }^{b}$ Department of Mathematical Science Bingham University, Karu, Nigeria
}

\begin{abstract}
In this study, a deterministic co-infection model of dengue virus and malaria fever is proposed. The disease free equilibrium point (DFEP) and the Basic Reproduction Number is derived using the next generation matrix method. Local and global stability of DFEP are analyzed. The results show that the DFEP is locally stable if $R_{0 d m}<1$ but may not be asymptotically stable. From the analysis of secondary data sourced from Kenyan region, the value of $R_{0 d m}$ computed is 19.70 greater than unity; this implies that dengue virus and malaria fever are endemic in the region. To identify the dominant parameter for the spread and control of the diseases and their co-infection, sensitivity analysis is investigated. From the numerical simulation using Maple 17, increase in the rate of recovery for co-infected individual contributes greatly in reducing dengue and malaria infections in the region. Decreasing either dengue or malaria contact rate also play a significant role in controlling the co-infection of dengue and malaria in the population. Therefore, the center for disease control and policy makers are expected to set out preventive measures in reducing the spread of both diseases and increase the approach of recovery for the co-infected individuals.
\end{abstract}

DOI: $10.46481 /$ jnsps.2021.196

Keywords: Co-infection, Dengue, Malaria, Stability Analysis, Sensitivity Analysis, Simulation.

Article History :

Received: 9 April 2021

Received in revised form: 30 April 2021

Accepted for publication: 5 May 2021

Published: 29 May 2021

(C)2021 Journal of the Nigerian Society of Physical Sciences. All rights reserved. Communicated by: B. J. Falaye

\section{Introduction}

The spread of mosquitoes borne diseases has gained concern globally in recent decades because of their recurring outbreaks. Millions of people die every year as a result of these infectious diseases and their control has increasingly become a complex issue [1]. Dengue virus and Malaria fever are common mosquitoes-borne diseases that have become a public health threat in the last few decades with high morbidity and mortality for many patients in various part of the world [2]. The world

\footnotetext{
${ }^{*}$ Corresponding author tel. no: +234(0)8130254488

Email address: ayubasolomona@gmail . com (Solomon Akyenyi Ayuba)
}

malaria report [3], estimated 229 million cases of malaria in 2019 compared to 228 millions cases in 2018, with 409000 deaths. $94 \%$ of the cases and deaths are reported from subSaharan Africa. Dengue is currently common in tropical and subtropical regions. The virus have four distinct stereotypes and are transmitted to human through bite of infected Aedes mosquitoes (aegyptic $\mathcal{E}$ albopictus) [4]. Dengue cases reported increased over 8 fold in the last two decades from 505430 cases in the year 2000 to 2.4 million in 2010 and to 4.2 million in 2019 [5]. While dengue is causing devastating impacts on the tropical and subtropical communities, malaria fever is endemic in some of these dengue affected regions there by drastically increases public health burden among the people in tropical 
communities living at risk of contracting both diseases concurrently. The two pathogens share similar geographical areas, and clinical distinction between them is difficult due to their overlapping symptoms. The work in [6], the researchers proposed a mathematical model to study the transmission dynamics of Zika and Malaria in malaria-endemic area. In Ref. [7] developed a novel mathematical model describing the co-infection dynamics of malaria and typhoid fever. [8] formulated A deterministic co-infection model between malaria and HIV in human population. Ref. [9] developed and analyzed the stability of disease free equilibrium point (DFEP) of a co-infection model between dengue virus and chikungunya in closed population. [10] developed a mathematical model for dengue-zika co-infection and carried out their synergistic relationship in the presence of prevention and treatment. Ref. [11] proposed a co-infection of altered vector infectivity and antibody- dependent enhancement of dengue-zika interplay. Ref. [12] formulated and analyzed a co-infection model of dengue fever and leptospirosis diseases. In [13], a deterministic model for dengue, malaria and typhoid triple co-infection was developed but limited only to the stability (Local and global) analysis. The authors in Ref. [14], developed a SEIR co-infection model of dengue and malaria but only established the local and global stability.

In this study, we propose a SIR-SI deterministic model of dengue virus and malaria co-infection and determine the stability analysis, sensitivity analysis and carryout numerical simulation for the co-infection model. The remainder of this paper is arranged as follows: In section 2, model descriptions, flow diagram (depicting the co-infection interactions) and the model formulation are presented. Section 3 is devoted to results and analysis; Invariant region, Disease free equilibrium point, Basic reproduction number, stability analysis, parameters estimation, sensitivity analysis and numerical simulation. Discussion of findings is presented in section 4. Finally, conclusions are drawn in section 5 and some possible directions for future studies are presented.

\section{Model Formulation}

${ }^{1}$ The data used in this study are secondarily sourced from $[7,15]$. In accordance with previous studies on mathematical model of dengue virus $[10,16,17,15]$ and malaria model $[19,20,21,22]$, we formulate a SIR-SI deterministic model of dengue and malaria co-infection. In this model, the total human population $N_{h}$ is partitioned into seven classes; susceptible human $S_{h}$, infected human with dengue virus $I_{h d}$, infected human with malaria $I_{h m}$, infected human with both dengue virus and malaria $I_{d m}$, recovery of infected human from dengue virus ,malaria fever and co-infected individuals are $R_{h d}, R_{h m}, R_{d m}$ respectively. The vectors population are subdivided into; susceptible dengue vector $S_{v d}$, dengue carrier vector $I_{v d}$, susceptible malaria vector $S_{v m}$ and malaria carrier vector $I_{v m}$. The recruitment rates for human, dengue and malaria vectors respectively, are $\Lambda_{h}, \Lambda_{d}$ and $\Lambda_{m}$. The recovery rate from dengue and malaria

\footnotetext{
${ }^{1}$ Stability and Sensitivity Analysis of Dengue-Malaria Co-infection Model
}

are $\sigma, \alpha$, transmission rate of dengue and malaria vectors to human per unit time are $\eta_{d}, \eta_{m}$, probability of dengue and malaria vectors to be infected are denoted by $\eta_{v d}, \eta_{v m}$ respectively. Recovered human from malaria become susceptible at $\gamma$ and acquired immunity $\rho$ rate. The co-infected individuals recover at the rate $\psi$; but those individuals either recover only from dengue and join $R_{h d}$ with probability of $q \psi$, or recover only from malaria and join $R_{h m}$ with probability of $\psi l(1-q)$, or recover from both diseases and join $R_{d m}$ with the probability of $\psi(1-l)(1-q)$. The human natural death rate denote $\mu_{h}$ while dengue and malaria vectors death rate are $\mu_{d}, \mu_{m}$ respectively. $\tau, \delta$ are dengue and malaria induced death rates while $\phi, \theta$ are dengue and malaria related death rates. The following assumptions are made to formulate the co-infection model: the total population is not constant, the susceptible rates are recruited through birth or immigration and the number increases from malaria recovered and co-infectious recovered individuals by losing their temporal immunity. Recovered individuals from dengue virus is permanent. Figure 2 shown the flow diagram for the interactions between dengue and malaria co-infection model in human population. The time dependent dynamical

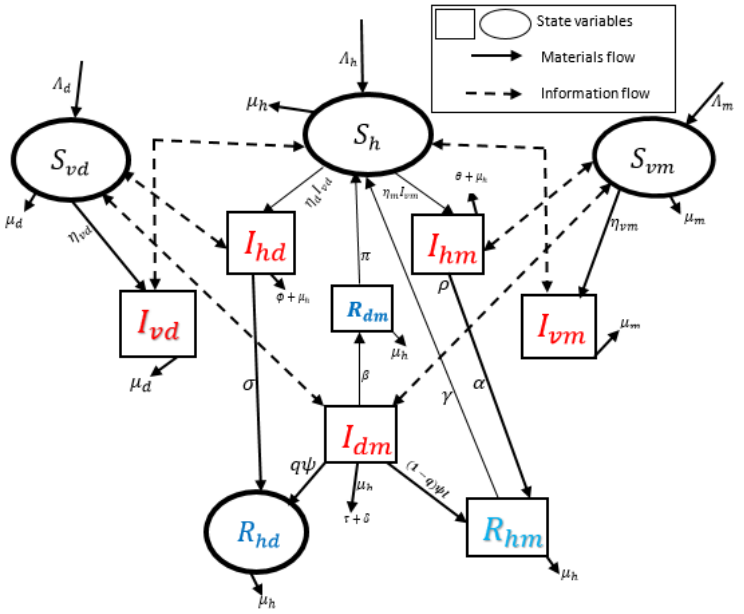

Figure 1. Flow diagram depicting Dengue virus and Malaria co-infection dynamics

system associated with the parameters interaction is shown as follows.

$$
\left\{\begin{array}{l}
S_{h}^{\prime}=\Lambda_{h}+\gamma R_{h m}+\pi R_{d m}-\frac{\left(\eta_{d} I_{v d}+\eta_{m} I_{v m}\right)}{N_{h}} S_{h}-\mu_{h} S_{h} \\
I_{h d}^{\prime}=\frac{\eta_{d} I_{v d}}{N_{h}} S_{h}-\frac{\eta_{m} I_{v m}}{N_{h}} I_{h d}-\left(\sigma+\tau+\mu_{h}+\phi\right) I_{h d} \\
I_{h m}^{\prime}=\frac{\eta_{m} I_{v m}}{N_{h}} S_{h}-\frac{\eta_{d} I_{v d}}{N_{h}} I_{h m}-\left(\alpha+\rho+\delta+\mu_{h}+\theta\right) I_{h m} \\
I_{d m}^{\prime}=\frac{\eta_{m} I_{v m}}{N_{h}} I_{h d}+\frac{\eta_{d} I_{v d}}{N_{h}} I_{h m}-\left(\psi+\mu_{h}+\theta+\phi\right) I_{d m} \\
R_{h d}^{\prime}=\sigma I_{h d}+q \psi I_{d m}-\mu_{h} R_{h d} \\
R_{h m}^{\prime}=\alpha I_{h m}+\psi l(1-q) I_{d m}-\left(\gamma+\mu_{h}\right) R_{h m} \\
R_{d m}^{\prime}=\psi(1-l)(1-q) I_{d m}-\left(\pi+\mu_{h}\right) R_{d m} \\
S_{v d}^{\prime}=\Lambda_{d}-\frac{\eta_{v d}\left(I_{h d}+I_{d m}\right)}{\left.N_{h}\right)} S_{v d}-\mu_{d} S_{v d} \\
I_{v d}^{\prime}=\frac{\eta_{v d} I_{h d}}{N_{h}} S_{v d}+\frac{\eta_{v v} I_{d m}}{N_{h}} S_{v d}-\mu_{d} I_{v d} \\
S_{v m}^{\prime}=\Lambda_{m}-\frac{\eta_{v m}\left(I_{h m}+I_{d m}\right)}{N_{h}} S_{v m}-\mu_{m} S_{v m} \\
I_{v m}^{\prime}=\frac{\eta_{v m} I_{h m}}{N_{h}} S_{v m}+\frac{\eta_{v m} I_{d m}}{N_{h}} S_{v m}-\mu_{m} I_{v m}
\end{array}\right.
$$


Table 1. Parameters description of Dengue and Malaria co-infection Model

\begin{tabular}{cc}
\hline Parameters & Description \\
\hline$\Lambda_{h}$ & Recruitment rate of Human Population \\
$\Lambda_{d}$ & Recruitment rate of Dengue Vectors \\
$\Lambda_{m}$ & Recruitment rate of Malaria Vectors \\
$\rho$ & Rate of human acquired immunity from Malaria \\
$\alpha$ & Rate of Human recovery from Malaria \\
$\sigma$ & Rate of Human recovery from Dengue \\
$\psi$ & Rate of human recovery from both Dengue and Malaria \\
$\gamma$ & Rate of Immunity warning for $R_{h m}$ to become susceptible \\
$\eta_{d}$ & Transmission rate of Dengue vectors to human per unit time \\
$\eta_{m}$ & Transmission rate of Malaria vectors to human per unit time \\
$\eta_{v d}$ & Probability for Dengue Vectors to be infected \\
$\eta_{v m}$ & Probability for Malaria parasite Vectors to be infected \\
$q \psi$ & Proportion of co-infected human recovery from Dengue only \\
$\psi l(1-q)$ & Proportion of co-infected human recovery from Malaria only \\
$\pi$ & Rate at which $R_{d m}$ become susceptible \\
$\tau$ & Disease induced death rate for human infected with Dengue \\
$\delta$ & Disease induced death rate for human infected with Malaria \\
$\phi$ & Dengue related death rate \\
$\mu_{h}$ & Malaria related death rate \\
$\mu_{d}$ & Natural death rate of humans \\
\hline & Natural death rate of Dengue vectors \\
\hline
\end{tabular}

\section{Results and Analysis}

\subsection{Invariant regions}

In this section, we obtain the bounded region of solution for the dengue-malaria model. The total human population is given by

$$
\begin{gathered}
N_{h}=S_{h}+I_{h d}+I_{h m}+I_{d m}+R_{h d}+R_{h m}+R_{d m}, \text { then } \\
N_{h}^{\prime}=S_{h}^{\prime}+I_{h d}^{\prime}+I_{h m}^{\prime}+R_{h d}^{\prime}+I_{d m}^{\prime}+R_{h m}^{\prime}+R_{d m}^{\prime} \\
\Longrightarrow N^{\prime}=\Lambda_{h}-\mu_{h} N_{h}
\end{gathered}
$$

Solving equation (3) as $t \rightarrow \infty$ yields

$$
D_{h}=\left\{\left(S_{h}, I_{h d}, I_{h m}, I_{d m}, R_{h d}, R_{h m}, R_{d m}\right) \in \mathfrak{R}^{7} ; 0 \leq N \leq \frac{\Lambda_{h}}{\mu_{h}}\right\}
$$

For the dengue vector population, if there is no spread of infection, then

$$
\begin{aligned}
& N_{d}^{\prime}=\Lambda_{d}-\mu_{d} N_{d} \\
& \quad D_{d}=\left\{\left(S_{v d}, I_{v d}\right) \in \mathfrak{R}^{2} ; N_{d} \leq \frac{\Lambda_{d}}{\mu_{d}}\right\}
\end{aligned}
$$

Similarly, for malaria vector population, we obtain

$$
\begin{aligned}
& N_{m}^{\prime}=\Lambda_{m}-\mu_{m} N_{m} \\
& \quad D_{m}=\left\{\left(S_{v m}, I_{v m}\right) \in \mathfrak{R}^{2} ; N_{m} \leq \frac{\Lambda_{m}}{\mu_{m}}\right\}
\end{aligned}
$$

Therefore, the feasible solution of dengue-malaria model is given by

$$
D=\left\{\left(D_{h} \times D_{d} \times D_{m}\right) \mathfrak{R}_{+}^{11}\right\}
$$

Thus, the solution of dengue-malaria model is bounded in $D$.

Theorem 3.1. If at $t=0$ and

$$
\begin{gathered}
\left\{S_{h}(0), I_{h d}(0), I_{h m}(0), I_{d m}(0), R_{h d}(0), R_{h m}(0), R_{d m}(0),\right. \\
\left.S_{v d}(0), I_{v d}(0), S_{v m}(0), I_{v m}(0)\right\} \geq 0,
\end{gathered}
$$

then the solution of dengue-malaria model are nonnegative at $t>0$.

\subsection{Existence of Disease Free Equilibrium Point}

2 To investigate the condition of existence of the disease free equilibrium point and also the asymptotic behaviour of the dengue-malaria co-infection model in this section, we will investigate whether the diseases die out or become endemic. This can only be addressed through the asymptotic behaviour of the diseases. This behaviour depends largely on the equilibrium point, that is time-independent solutions of the system. Since these solutions are independent of time, we set the left hand side of system (1) to zero. $S_{h}^{\prime}=I_{h d}^{\prime}=I_{h m}^{\prime}=I_{d m}^{\prime}=R_{h d}^{\prime}=R_{h m}^{\prime}=$ $R_{d m}^{\prime}=0$ and $S_{v d}^{\prime}=I_{v d}^{\prime}=S_{v m}=I_{v m}^{\prime}=0$.

${ }^{2}$ Stability and Sensitivity Analysis of Dengue-Malaria Co-infection Model 
Thus, the equilibrium point is given by

$$
\begin{gathered}
E_{0 d m}=\left[S_{h}(0), I_{h d}(0), I_{h m}(0), I_{d m}(0), R_{h d}(0), R_{h m}(0),\right. \\
\left.R_{d m}(0), S_{v d}(0), I_{v d}(0), S_{v m}(0), I_{v m}(0)\right] \\
=\left[\frac{\Lambda_{h}}{\mu_{h}}, 0,0,0,0,0,0, \frac{\Lambda_{d}}{\mu_{d}}, 0, \frac{\Lambda_{m}}{\mu_{m}}, 0\right]
\end{gathered}
$$

\subsection{Basic Reproduction Number $R_{0 d m}$}

The linear stability of the equilibrium point $E_{0 d m}$ is established using next generation matrix method on system (1) to obtain the threshold behavior $R_{0 d m}$. Hence, we introduce two matrices; matrix $\mathbf{A}$ for rates of new infection and $\mathbf{B}$ is the transfer rate of in or out of a compartment. Taking the partial derivative of the right hand side of (1) at DFEP with respect to $I_{h d}, I_{h m}, I_{d m}, I_{v d}, I_{v m}$, we obtain

$$
\begin{aligned}
A & =\left(\begin{array}{ccccc}
0 & 0 & 0 & \frac{\eta_{d} \Lambda_{h}}{\mu_{h} N_{h}} & 0 \\
0 & 0 & 0 & 0 & \frac{\eta_{m} \Lambda_{h}}{\mu_{h} N_{h}} \\
0 & 0 & 0 & 0 & 0 \\
\frac{\eta_{v d} \Lambda_{d}}{\mu_{d} N_{h}} & 0 & \frac{\eta_{v d} \Lambda_{d}}{\mu_{d} N_{h}} & 0 & 0 \\
0 & \frac{\eta_{v m} \Lambda_{m}}{\mu_{m} N_{h}} & \frac{\eta_{v m} \Lambda_{m}}{\mu_{m} N_{h}} & 0 & 0
\end{array}\right) \\
B & =\left(\begin{array}{ccccc}
-\kappa_{1} & 0 & 0 & 0 & 0 \\
0 & -D_{T} & 0 & 0 & 0 \\
0 & 0 & -\kappa_{2} & 0 & 0 \\
0 & 0 & 0 & -\mu_{d} & 0 \\
0 & 0 & 0 & 0 & -\mu_{m}
\end{array}\right) \\
\therefore B^{-1} & =\left[\begin{array}{ccccc}
-\frac{1}{\kappa_{1}} & 0 & 0 & 0 & 0 \\
0 & -\frac{1}{D_{T}} & 0 & 0 & 0 \\
0 & 0 & -\frac{1}{\kappa_{2}} & 0 & 0 \\
0 & 0 & 0 & -\frac{1}{\mu_{d}} & 0 \\
0 & 0 & 0 & 0 & -\frac{1}{\mu_{m}}
\end{array}\right]
\end{aligned}
$$

where $\kappa_{1}=\left(\sigma+\tau+\mu_{h}+\phi\right), \kappa_{2}=\left(\psi+\mu_{h}+\theta+\phi\right), D_{T}=\left(\alpha+\rho+\delta+\mu_{h}+\theta\right)$ and $\beta=\psi(1-l)(1-q)$ from equation $(1)$. The basic reproduction number $R_{0 d m}$ of dengue-malaria co-infection model is the number of secondary infections of dengue or malaria in the population due to a single dengue or malaria infective individual. The reproduction number is the spectral radius of $A B^{-1}$ defined as $R_{0 d m}:=p\left(A B^{-1}\right)$, and is given by

$$
R_{0 d m}=\max \left\{\sqrt{\frac{\eta_{d} \eta_{v d} \Lambda_{d} \Lambda_{h}}{\mu_{h} \mu_{d}^{2} \kappa_{1} N_{h}^{2}}}, \sqrt{\frac{\eta_{m} \eta_{v m} \Lambda_{m} \Lambda_{h}}{\mu_{m}^{2} \mu_{h} D_{T} N_{h}^{2}}}\right\}
$$

\subsubsection{Local stability of disease free equilibrium point}

${ }^{3}$ The Jacobian matrix $J_{0 d m}$ of dengue-malaria model (1) at $E_{0 d m}$ is obtained as seen in matrix (8).

$$
\left[\begin{array}{ccccccccccc}
-\mu_{h} & 0 & 0 & 0 & 0 & \gamma & \pi & 0 & \frac{-\eta_{d} \Lambda_{h}}{\mu_{h} N_{h}} & 0 & \frac{-\eta_{m} \Lambda_{h}}{\mu_{h} N_{h}} \\
0 & -\kappa_{1} & 0 & 0 & 0 & 0 & 0 & 0 & \frac{\eta_{d} \Lambda_{h}}{\mu_{h} N_{h}} & 0 & 0 \\
0 & 0 & -D_{T} & 0 & 0 & 0 & 0 & 0 & 0 & 0 & \frac{\eta_{m} \Lambda_{h}}{\mu_{h} N_{h}} \\
0 & 0 & 0 & -\kappa_{2} & 0 & 0 & 0 & 0 & 0 & 0 & 0 \\
0 & \sigma & 0 & q \psi & -\mu_{h} & 0 & 0 & 0 & 0 & 0 & 0 \\
0 & 0 & \rho & \psi l(1-q) & 0 & \left(-\gamma-\mu_{h}\right) & 0 & 0 & 0 & 0 & 0 \\
0 & 0 & 0 & \beta & 0 & 0 & \left(-\pi-\mu_{h}\right) & 0 & 0 & 0 & 0 \\
0 & \frac{-\eta_{v d} \Lambda_{d}}{\mu_{d} N_{h}} & 0 & \frac{-\eta_{v d} \Lambda_{d}}{\mu_{d} N_{h}} & 0 & 0 & 0 & -\mu_{d} & 0 & 0 & 0 \\
0 & \frac{\eta_{v d} \Lambda_{d}}{\mu_{d} N_{h}} & 0 & \frac{\eta_{v d} \Lambda_{d}}{\mu_{d} N_{h}} & 0 & 0 & 0 & 0 & -\mu_{d} & 0 & 0 \\
0 & 0 & \frac{-\eta_{v m} \Lambda_{m}}{\mu_{m} N_{h}} & \frac{\eta_{v m} \Lambda_{m}}{\mu_{m} N_{h}} & 0 & 0 & 0 & 0 & 0 & -\mu_{m} & 0 \\
0 & 0 & \frac{\eta_{v m} \Lambda_{m}}{\mu_{m} N_{h}} & \frac{\eta_{v m} \Lambda_{m}}{\mu_{m} N_{h}} & 0 & 0 & 0 & 0 & 0 & 0 & -\mu_{m}
\end{array}\right]
$$

Theorem 3.2. The disease free equilibrium $E_{0} d m$ is locally asymptotically stable if $R_{0 d m}<1$ and unstable if $R_{0 d m}>1$.

\footnotetext{
${ }^{3}$ Stability and Sensitivity Analysis of Dengue-Malaria Co-infection Model
} 
Proof 3.1. . The local stability of $E_{0 d m}$ is establish by the Jacobian matrix (8) at $E_{0 d m}$. The characteristic polynomial of $J_{0 d m}$ is determine by

$$
\begin{aligned}
& \operatorname{det}\left(J_{0 d m}-t I\right)=\left(-\mu_{h}-t\right) \\
& \times\left(-\mu_{h}-t\right) \times\left(-\gamma-\mu_{h}-t\right) \\
& \times\left(-\pi-\mu_{h}-t\right)\left(-\mu_{d}-t\right) \\
& \times\left(-\mu_{m}-t\right) \times \operatorname{det}\left(\hat{J}_{0 d m}-t I\right)=0
\end{aligned}
$$

where $\hat{J}_{0 d m}$ is given by

$$
\hat{J}_{0 d m}=\left[\begin{array}{ccccc}
-\kappa_{1} & 0 & 0 & \frac{\eta_{d} \Lambda_{h}}{\mu_{h} N_{h}} & 0 \\
0 & -D_{T} & 0 & 0 & \frac{\eta_{m} \Lambda_{h}}{\mu_{h} N_{h}} \\
0 & 0 & -\kappa_{2} & 0 & 0 \\
\frac{\eta_{v} d \Lambda_{d}}{\mu_{d} N_{h}} & 0 & \frac{\eta_{v d} \Lambda_{d}}{\mu_{d} N_{h}} & -\mu_{d} & 0 \\
0 & \frac{\eta_{v} m \Lambda_{m}}{\mu_{m} N_{h}} & \frac{\eta_{v} m \Lambda_{m}}{\mu_{m} N_{h}} & 0 & -\mu_{m}
\end{array}\right]
$$

Using the properties of determinant, we obtain

$$
\begin{aligned}
& \operatorname{det}\left(\hat{J}_{0 d m}-I t\right)=\operatorname{det}\left[\begin{array}{ccccc}
-D_{T}-t & 0 & \frac{\eta_{m} \Lambda_{h}}{\mu_{h} N_{h}} & 0 & 0 \\
0 & -\kappa_{2}-t & 0 & 0 & 0 \\
\frac{\eta_{v m} \Lambda_{m}}{\mu_{h} N_{h}} & \frac{\eta_{v m} \Lambda_{m}}{\mu_{h} N_{h}} & -\mu_{m}-t & 0 & 0 \\
0 & \frac{\eta_{v d} \Lambda_{d}}{\mu_{h} N_{h}} & 0 & -\mu_{d}-t & \frac{\eta_{v d} \Lambda_{d}}{\mu_{h} N_{h}} \\
0 & 0 & 0 & -\frac{\eta_{d} \Lambda_{h}}{\mu_{h} N_{h}}-t & -\kappa_{1}-t
\end{array}\right] \\
& \operatorname{det}\left(\begin{array}{ccc}
-D_{T}-t & 0 & \frac{\eta_{m} \Lambda_{h}}{\mu_{h} N_{h}} \\
0 & -\kappa_{2}-t & 0 \\
\frac{\eta_{v m} \Lambda_{m}}{\mu_{m} N_{h}} & \frac{\eta_{v m} \Lambda_{m}}{\mu_{m} N_{h}} & -\mu_{m}-t
\end{array}\right) \times \operatorname{det}\left(\begin{array}{cc}
-\mu_{d}-t & -\frac{\eta_{v d} \Lambda_{d}}{\mu_{d} N_{h}} \\
\frac{\eta_{d} \Lambda_{h}}{\mu_{h} N_{h}} & -\kappa_{1}-t
\end{array}\right)=0
\end{aligned}
$$

The five eigenvalues of $J_{0 d m}$ are $\left(-\mu_{h}-t\right) \times\left(-\mu_{h}-t\right) \times(-\gamma-$ $\left.\mu_{h}-t\right) \times\left(-\mu_{d}-t\right) \times\left(-\mu_{m}-t\right)=0$ and the other five eigenvalues are obtained from the solution of matrix equation (9) by

$$
\begin{aligned}
\operatorname{det}\left(\begin{array}{ccc}
-D_{T}-t & 0 & \frac{\eta_{m} \Lambda_{h}}{\mu_{h} N_{h}} \\
0 & -\kappa_{2}-t & 0 \\
\frac{\eta_{v m} \Lambda_{m}}{\mu_{m} N_{h}} & \frac{\eta_{v m} \Lambda_{m}}{\mu_{m} N_{h}} & -\mu_{m}-t
\end{array}\right) & =0 \\
\operatorname{det}\left(\begin{array}{cc}
-\mu_{d}-t & -\frac{\eta_{v d} \Lambda_{d}}{\mu_{d} N_{h}} \\
\frac{\eta_{d} \Lambda_{h}}{\mu_{h} N_{h}} & -\kappa_{1}-t
\end{array}\right) & =0
\end{aligned}
$$

The above determinant becomes

$$
\begin{gathered}
t^{3}-\left(D_{T}+\kappa_{2}+\mu_{m}\right) t^{2} \\
-\left(\kappa_{2}\left(D_{T}+\left(D_{T}+\kappa_{2}\right)+\left(1-R_{0 m}^{2}\right) D_{T} \mu_{m}\right) t\right. \\
+\left(\kappa_{2}-R_{0 m}^{2}\right) D_{T} \mu_{m}=0 \\
t^{2}+\left(\mu_{d}+\kappa_{1}\right) t+\left(1-R_{0 d}^{2}\right) \mu_{d} \kappa_{1}=0
\end{gathered}
$$

The above eigenvalues of equation (10) and (11) are also negative. Therefore, the disease free equilibrium point are locally asymptotically stable iff $R_{0 d}<1$ and $R_{0 m}<1$.

\subsubsection{Global stability of disease free equilibrium point}

4 The global asymptotic stability of the DFEP is investigated using Carlos Castillo-Chavez conditions as described in [23]. From the co-infection model (1), we define the time dependent derivatives by

$$
\begin{array}{r}
X^{\prime}=F(X, Z) \\
Z^{\prime}=G(X, Z), G(X, 0)=0
\end{array}
$$

Where $X=\left(S_{h}, R_{h d}, R_{h m}, R_{d m}, S_{v d}, S_{v m}\right)$ and $Z=\left(I_{h d}, I_{h m}, I_{d m}, I_{v d}, I_{v m}\right)$ denote uninfected and infected populations respectively. To guarantee the global asymptotic stability, the following conditions must be satisfied.

(a) $X^{\prime}=F(X, 0) ; X^{*}$ is globally stable

(b) $G(X, Z)=D_{z} G\left(X^{*}, 0\right) Z-\hat{G}(X, Z), \hat{G}(X, Z) \geq 0 \forall X, Z \in$ $\Omega$

Theorem 3.3. The equilibrium point $E_{0 d m}=\left(X^{*}, 0\right)$ of system (1) is globally asymptotically stable if $R_{0 d m} \leq 1$ and the conditions (a), (b) are satisfied.

Proof: $F(X, Z)$ and $G(X, Z)$ is given by

$$
F(X, Z)=\left[\begin{array}{c}
\Lambda_{h}+\gamma R_{h m}+\pi R_{d m}-\frac{\eta_{d} I_{v d}+\eta_{m} I_{v m}}{N_{h}} S_{h}-\mu_{h} S_{h} \\
\sigma R_{h d}+q \psi I_{d m}-\mu_{h} R_{h d} \\
\rho R_{h m}+(1-q \psi) I_{h m}-\left(\gamma+\mu_{h}\right) R_{h} m \\
\beta I_{d m}-\left(\pi+\mu_{h}\right) R_{d m} \\
\Lambda_{d}-\frac{\eta_{v d}\left(I_{h d}+I_{d m}\right)}{N_{h}} S_{v d}-\mu_{d} S_{v d} \\
\Lambda_{m}-\frac{\eta_{v m}\left(I_{h m}+I_{d m}\right)}{N_{h}} S_{v m}-\mu_{m} S_{v m}
\end{array}\right]
$$

$$
G(X, Z)=\left[\begin{array}{c}
\frac{\eta_{d} I_{v d}}{N_{h}} S_{h}-\frac{\eta_{m} I_{v m}}{N_{h}} I_{h d}-\left(\sigma+\tau+\mu_{h}+\phi\right) I_{h d} \\
\frac{\eta_{m} I_{v m}}{N_{h}} S_{h}-\frac{\eta_{d} v_{v d}}{N_{h} I_{h m}-\left(\alpha+\rho+\delta+\mu_{h}+\theta\right) I_{h m}} \\
\frac{\eta_{m} I_{v m}}{N_{h}} I_{h d}+\frac{\eta_{d} I_{v d}}{N_{h}} I_{h m}-\left(\psi+\mu_{h}+\theta+\phi\right) I_{d m} \\
\frac{\eta_{v v} I_{h d}}{N_{h}} S_{v d}+\frac{\eta_{v v} I_{d m}}{N_{m}} S_{v d}-\mu_{d} I_{v d} \\
\frac{\eta_{v v} I_{h m}}{N_{h}} S_{v m}+\frac{\eta_{v m} l_{d m}}{N_{h}} S_{v m}-\mu_{m} I_{v m}
\end{array}\right]
$$

For $X^{\prime}=F(X, 0)$, system (1) is reduced to

$$
X^{\prime}=\left\{\begin{array}{l}
S_{h}^{\prime}=\Lambda_{h}+\pi R_{d m}+\gamma R_{h m}-\mu_{h} S_{h} \\
S_{v d^{\prime}}=\Lambda_{d}-\mu_{d} S_{v d} \\
S_{v m}=\Lambda_{m}-\mu_{m} S_{v m} \\
\text { with } X^{*}=\left(\frac{\Lambda_{h}}{\mu_{h}}, \frac{\Lambda_{d}}{\mu_{d}}, \frac{\Lambda_{m}}{\mu_{m}}\right)
\end{array}\right.
$$

Given $G(X, Z)=D_{z} G\left(X^{*}, 0\right) Z-\hat{G}(X, Z), \hat{G}(X, Z) \geq 0$

$$
G\left(X^{*}, 0\right)=\left[\begin{array}{ccccc}
-\kappa_{1} & 0 & 0 & \frac{\eta_{d} \Lambda_{h}}{\mu_{h} N_{h}} & 0 \\
0 & -D_{T} & 0 & 0 & \frac{\eta_{m} \Lambda_{h}}{\mu_{h} N_{h}} \\
0 & 0 & -\kappa_{2} & 0 & 0 \\
\frac{\eta_{v d} \Lambda_{d}}{\mu_{d} N_{h}} & 0 & \frac{\eta_{v d} \Lambda_{d}}{\mu_{d} N_{h}} & -\mu_{d} & 0 \\
0 & \frac{\eta_{v m} \Lambda_{m}}{\mu_{m} N_{h}} & \frac{\eta_{v m} \Lambda_{m}}{\mu_{m} N_{h}} & 0 & -\mu_{m}
\end{array}\right]
$$




$$
\begin{aligned}
& D_{z} G\left(X^{*}, 0\right) Z=\left[\begin{array}{ccccc}
-\kappa_{1} & 0 & 0 & \frac{\eta_{d} \Lambda_{h}}{\mu_{h} N_{h}} & 0 \\
0 & -D_{T} & 0 & 0 & \frac{\eta_{m} \Lambda_{h}}{\mu_{h} N_{h}} \\
0 & 0 & -\kappa_{2} & 0 & 0 \\
\frac{\eta_{v d} \Lambda_{d}}{\mu_{d} N_{h}} & 0 & \frac{\eta_{v d} \Lambda_{d}}{\mu_{d} N_{h}} & -\mu_{d} & 0 \\
0 & \frac{\eta_{v m} \Lambda_{m}}{\mu_{m} N_{h}} & \frac{\eta_{v m} \Lambda_{m}}{\mu_{m} N_{h}} & 0 & -\mu_{m}
\end{array}\right] \\
& \times\left(\begin{array}{c}
I_{h d} \\
I_{h m} \\
I_{d m} \\
I_{v d} \\
I_{v m}
\end{array}\right)=\left[\begin{array}{c}
-\kappa_{1} I_{h d}+\frac{\eta_{d} \Lambda_{h}}{\mu_{h} N_{h}} I_{v d} \\
-D_{T} I_{h m}+\frac{\eta_{m} \Lambda_{h}}{\mu_{h} N_{h}} I_{v m} \\
-\kappa_{2} I_{d m} \\
\frac{\eta_{v d} \Lambda_{d}}{\mu_{d} N_{h}} I_{h d}+\frac{\eta_{v d} \Lambda_{d}}{\mu_{d} N_{h}} I_{d m}-\mu_{d} I_{v d} \\
\frac{\eta_{v m} \Lambda_{m}}{\mu_{m} N_{h}} I_{h m}+\frac{\eta_{v m} \Lambda_{m}}{\mu_{m} N_{h}} I_{d m}-\mu_{d} I_{v m}
\end{array}\right] \\
& \hat{G}(X, Z)=\left[\begin{array}{l}
\hat{G_{1}}(X, Z) \\
\hat{\hat{G}_{2}}(X, Z) \\
\hat{G_{3}}(X, Z) \\
\hat{G_{4}}(X, Z) \\
\hat{G_{5}}(X, Z)
\end{array}\right]=\left[\begin{array}{c}
\frac{\eta_{d} I_{v d}}{N_{h}}\left(\frac{\Lambda_{h}}{\mu_{h}}-S_{h}\right)+\frac{\eta_{m} I_{v m} I_{h d}}{N_{h}} \\
\frac{\eta_{m} I_{v m}}{N_{h}}\left(\frac{\Lambda_{h}}{\mu_{h}}-S_{h}\right)+\frac{\eta_{d} I_{v d} I_{h m}}{N_{h}} \\
-\left(\frac{\eta_{m} I_{v m} I_{h d}}{N_{h}}+\frac{\eta_{d} I_{v d} I_{h m}}{N_{h}}\right) \\
\frac{\eta_{v d}}{N_{h}}\left(\frac{\Lambda_{d}}{\mu_{d}}-S_{v d}\right)\left(I_{h d}+I_{d m}\right) \\
\frac{\eta_{v m}}{N_{h}}\left(\frac{\Lambda_{m}}{\mu_{m}}-S_{v m}\right)\left(I_{h m}+I_{d m}\right)
\end{array}\right]
\end{aligned}
$$

Since $\hat{G}_{3}(X, Z)<0$ in equation (16) and condition (b) requires $\hat{G}(X, Z) \geq 0$. Hence, condition (b) is not met as $\hat{G}(X, Z)<$ 0 for all $X, Z \in \Omega$. Thus, it implies that the DEFP may not be globally asymptotically stable if $R_{0 d m}<1$. Therefore, the endemic equilibrium exist with DFEP if $R_{\text {odm }}<1$. Whence, we can deduced that the dengue-malaria model exhibits backward bifurcation when the basic reproduction number $R_{0 d m}=1$.

\subsection{Parameters Estimation and Sensitivity Analysis}

\subsubsection{Parameters estimation and initial value}

5 The parameters in Table 2 are obtained (or estimated) in line with the work of $[7,15]$, from Kenyan region where malaria and dengue virus are said to be endemic. Conservatively, the following initial values are estimated. The total human population is estimated to be $52,000,000$ and the susceptible human are assumed to be $25,000,000$ which is about half of the population at the onset of the diseases. For vectors population, $10,000,000$ is assumed to be susceptible malaria mosquitoes with 2,000, 000 malaria carrier mosquitoes. Dengue susceptible mosquitoes are estimated to 5,000,000 and 100,000 for dengue carrier mosquitoes. Therefore, the initial infected human with malaria is estimated to be 10,000 and infected human with dengue estimate is 5000 .

\subsection{Sensitivity analysis of the model}

In order to identify the dominant parameter for the spread and control of dengue and malaria infections in the population, we performed the sensitivity analysis. As described in Carlos

\footnotetext{
${ }^{5}$ Stability and Sensitivity Analysis of Dengue-Malaria Co-infection Model
}

Table 2. Parameters values of dengue-malaria co-infection model

\begin{tabular}{c|c|c}
\hline Parameter & Value/day & Source \\
\hline$\Lambda_{h}$ & 467 & {$[7]$} \\
$\mu_{h}$ & 0.00004 & calculated \\
$\Lambda_{d}$ & 221056.75 & estimated \\
$\eta_{d}$ & 0.000451 & estimated \\
$\eta_{v d}$ & 0.13502 & estimated \\
$\sigma$ & 0.035 & estimated \\
$\pi$ & 0.003 & estimated \\
$\tau$ & 0.0245 & estimated \\
$\phi$ & 0.00023 & estimated \\
$\mu_{d}$ & 0.00005 & calculated \\
$\eta_{m}$ & 0.000408 & {$[7]$} \\
$\eta_{v m}$ & 0.15096 & {$[7]$} \\
$\gamma$ & $0.06[0,1]$ & {$[7]$} \\
$\alpha$ & 0.038 & {$[7]$} \\
$\rho$ & 0.37 & {$[7,15]$} \\
$\delta$ & 0.0019 & {$[7]$} \\
$\theta$ & 0.00025 & estimated \\
$\mu_{m}$ & 0.00005 & calculated \\
\hline
\end{tabular}

Castillo-Chavez [23], the sensitivity index of $R_{0 d m}$ with a parameter say $\beta$ is expressed as

$$
\Upsilon_{\beta}^{R_{0 d m}}=\frac{\partial R_{o d m}}{\partial \beta} \times \frac{\beta}{R_{0 d m}}
$$

Since $R_{o d m}$ is defined by

$$
R_{0 d m}=\left\{\sqrt{\frac{\eta_{d} \eta_{v d} \Lambda_{d} \Lambda_{h}}{\mu_{h} \mu_{d}^{2} \kappa_{1} N_{h}^{2}}}, \sqrt{\frac{\eta_{m} \eta_{v m} \Lambda_{m} \Lambda_{h}}{\mu_{m}^{2} \mu_{h} D_{T} N_{h}^{2}}}\right\}
$$

Therefore, we evaluate the sensitivity index of $R_{0 d}$ and $R_{o m}$ separately as follows: ${ }^{6}$

$$
\begin{array}{r}
\Upsilon_{\eta_{d}}^{R_{0 d}}=\frac{\partial R_{0 d}}{\partial \eta_{d}} \times \frac{\eta_{d}}{R_{0 d}}=\frac{1}{2}>0 \\
\Upsilon_{\eta_{v d}}^{R_{0 d}}=\frac{\partial R_{0 d}}{\partial \eta_{v d}} \times \frac{\eta_{v d}}{R_{0 d}}=\frac{1}{2}>0 \\
\Upsilon_{\sigma}^{R_{0 d}}=\frac{\partial R_{0 d}}{\partial \sigma} \times \frac{\sigma}{R_{0 d}}=-\frac{\sigma}{2 \kappa_{1}}<0 \\
\Upsilon_{\tau}^{R_{0 d}}=\frac{\partial R_{0 d}}{\partial \tau} \times \frac{\tau}{R_{0 d}}=-\frac{\tau}{2 \kappa_{1}}<0 \\
\Upsilon_{\phi}^{R_{0 d}}=\frac{\partial R_{0 d}}{\partial \phi} \times \frac{\phi}{R_{0 d}}=-\frac{\phi}{2 \kappa_{1}}<0 \\
\Upsilon_{\mu_{d}}^{R_{0 d}}=\frac{\partial R_{0 d}}{\partial \mu_{d}} \times \frac{\mu_{d}}{R_{0 d}}=-1<0 \\
\Upsilon_{\mu_{h}}^{R_{0 d}}=\frac{\partial R_{0 d}}{\partial \mu_{h}} \times \frac{\mu_{h}}{R_{0 d}}=-\frac{\sigma+\tau+2 \mu_{h}+\phi}{2 \kappa_{1}}<0
\end{array}
$$

${ }^{6}$ Stability and Sensitivity Analysis of Dengue-Malaria Co-infection Model 


$$
\begin{array}{r}
\Upsilon_{\eta_{m}}^{R_{0 m}}=\frac{\partial R_{0 m}}{\partial \eta_{m}} \times \frac{\eta_{m}}{R_{0 m}}=\frac{1}{2}>0 \\
\Upsilon_{\eta_{v m}}^{R_{0 m}}=\frac{\partial R_{0 m}}{\partial \eta_{v m}} \times \frac{\eta_{v m}}{R_{0 m}}=\frac{1}{2}>0 \\
\Upsilon_{\alpha}^{R_{0 m}}=\frac{\partial R_{0 m}}{\partial \alpha} \times \frac{\alpha}{R_{0 m}}=-\frac{\alpha}{2 D_{T}}<0 \\
\Upsilon_{\rho}^{R_{0 m}}=\frac{\partial R_{0 m}}{\partial \rho} \times \frac{\rho}{R_{0 m}}=-\frac{\rho}{2 D_{T}}<0 \\
\Upsilon_{\delta}^{R_{0 m}}=\frac{\partial R_{0 m}}{\partial \delta} \times \frac{\delta}{R_{0 m}}=-\frac{\delta}{2 D_{T}}<0 \\
\Upsilon_{\theta}^{R_{0 m}}=\frac{\partial R_{0 m}}{\partial \theta} \times \frac{\theta}{R_{0 m}}=-\frac{\theta}{2 D_{T}}<0 \\
\Upsilon_{\mu_{m}}^{R_{0 m}}=\frac{\partial R_{0 m}}{\partial \mu_{m}} \times \frac{\mu_{m}}{R_{0 m}}=-\frac{\alpha+\rho+\delta+2 \mu_{m}+\theta}{2 D_{T}}<0
\end{array}
$$

is kept constant. Hence, this can be use by policy makers to combat the disease.

The parameters with positive sensitivity indices are $\eta_{d}, \eta_{v d}, \eta_{m}, \eta_{v m}$ and the negative indices includes $\sigma, \tau, \phi, \mu_{d}, \alpha, \rho, \delta, \theta, \mu_{m}$. The positive sign parameters have great influence in the spread of the diseases and their co-infection in the region. Whereas, the parameters with negative sign have potential influence on the control of the spread of dengue, malaria and their co-infection. Hence, the center for disease control is expected to make policies and control measures in this regard to combat dengue, malaria and their co-infection in an endemic region.

\subsection{Numerical Simulations}

\subsubsection{Effect of malaria recovery rate $(\alpha)$ on infectious $\left(I_{h m}\right)$ population}

As seen in Figure 2, it is shown that $\alpha$ plays a significant influence in decreasing malaria infection. When the value of $\alpha$ increases from 0.038 to 1 , the infectious population due to malaria decreased, where the contact rate $\eta_{m}$ is kept constant.

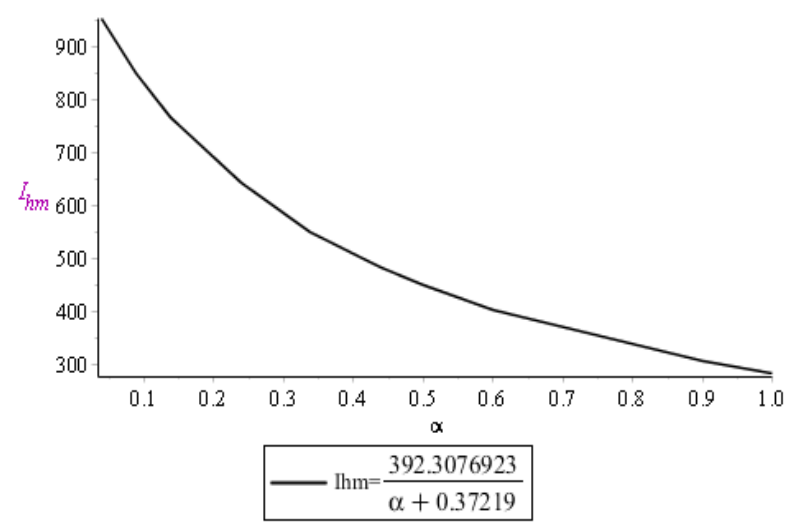

Figure 2. Effect of malaria recovery rate on infectious population

\subsubsection{Effect of dengue recovery rate $(\sigma)$ on infectious $\left(I_{h d}\right)$ population}

In Figure 3, as the value of $\sigma$ varies from 0.035 to 0.99 , the number of dengue infection decreases when the contact rate $\eta_{d}$

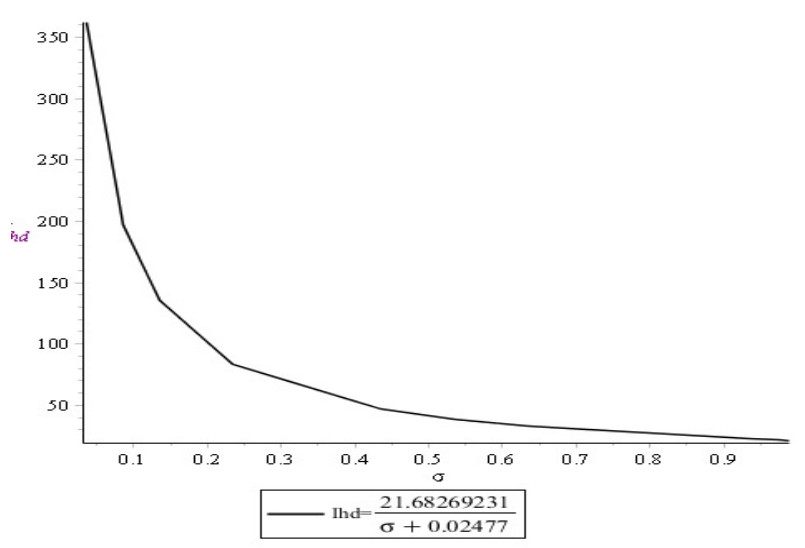

Figure 3. Effect of dengue recovery rate on infectious population

\subsubsection{Effect of dengue contact rate $\left(\eta_{d}\right)$ on co-infectious $\left(I_{d m}\right)$ population}

In Figure 4, the contact rate of dengue $\eta_{d}$ varies from 0.000451 to 0.040451 , the number of co-infectious population increases as the recovery rate is kept constant. Thus, the center for disease control and policy makers are expected to apply vector control measures and mechanism to reduce the expansion of co-infection in the region.

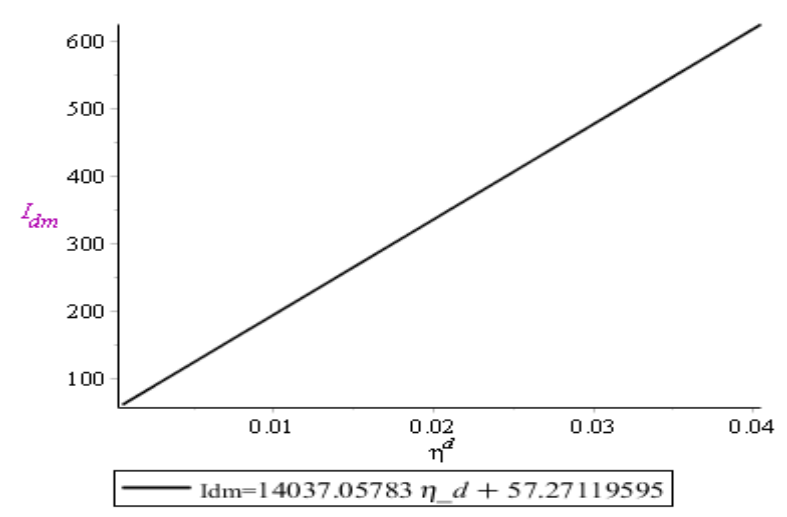

Figure 4. Effect of dengue contact rate on co-infectious population

\subsubsection{Effect of dengue-malaria recovery rate $(\psi)$ on co-infectious $\left(I_{d m}\right)$ population}

The recovery rate described in dengue-malaria model is either the individual recovery from dengue only, recovery from malaria only or both dengue and malaria infections. As shown in Figure 5, increasing $\psi$ play a significant role in reducing both dengue and malaria infections in the region. 
Table 3. Parameters value and sensitivity indices

\begin{tabular}{c|c|c}
\hline Parameter & Sensitivity indice & Sensitivity index \\
\hline $\mathfrak{R}_{0 d}$ & Basic reproduction number of dengue & - \\
$\mu_{h}$ & $-\mathrm{ve}$ & -0.001787 \\
$\eta_{d}$ & $+\mathrm{ve}$ & +0.5 \\
$\eta_{v d}$ & $+\mathrm{ve}$ & +0.5 \\
$\sigma$ & $-\mathrm{ve}$ & -0.001046 \\
$\tau$ & $-\mathrm{ve}$ & -0.000732 \\
$\phi$ & $-\mathrm{ve}$ & -0.000007 \\
$\mu_{d}$ & $-\mathrm{ve}$ & -1 \\
$\mathfrak{R}_{0 m}$ & Basic reproduction number of malaria & - \\
$\eta_{m}$ & $+\mathrm{ve}$ & +0.5 \\
$\eta_{v m}$ & $+\mathrm{ve}$ & +0.5 \\
$\alpha$ & $-\mathrm{ve}$ & -0.007794 \\
$\rho$ & $-\mathrm{ve}$ & 0.075885 \\
$\delta$ & $-\mathrm{ve}$ & -0.00049 \\
$\theta$ & $-\mathrm{ve}$ & -0.00005 \\
$\mu_{m}$ & $-\mathrm{ve}$ & -1 \\
\hline
\end{tabular}

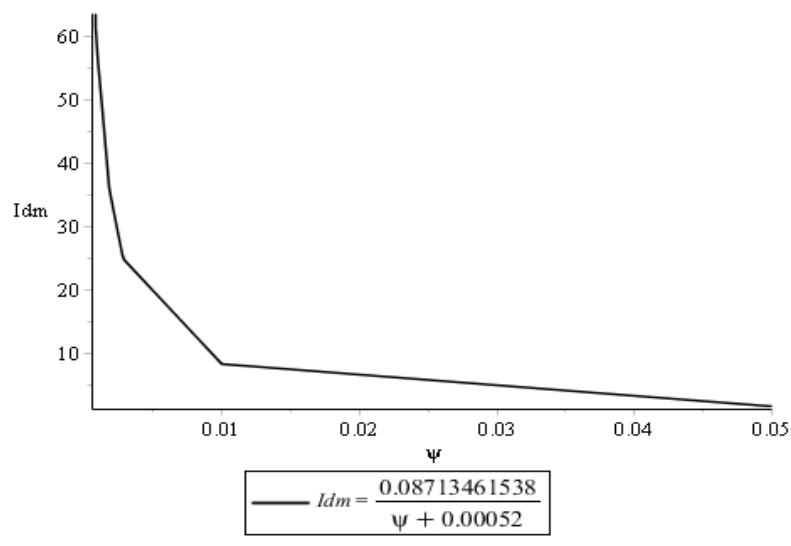

Figure 5. Effect of recovery rate on co-infection population

\section{Discussion}

In this paper, we develop a deterministic mathematical model that studies the dynamics of dengue virus and malaria fever in an endemic stage. Base on the qualitative and numerical analysis of the data sourced from $[7,15]$ with conservative estimates, the results depict some interesting insights into the underlying relationship between dengue virus and malaria fever and provide information that are useful to combat the diseases. The qualitatively analysis of the model shows that there is a bounded invariant region where the model is mathematical and epidemiological well posed. The basic reproduction number of the model was derived using the next generation matrix method. Stability and sensitivity analysis of the disease free equilibrium point (DFEP) were established. The result shows that the DFEP is locally stable if $R_{0 d m}<1$ but may not be asymptotically stable. Therefore, the endemic equilibrium exist when $R_{\text {odm }}<1$ with DFEP and this implies that the model undergoes backward bifurcation. We demonstrated numerically using Maple 17 , the effects of basic parameters for the spread and control of dengue and malaria co-infection. From the results, we conclude that an increase in dengue and malaria recovery rates plays a great role in reducing dengue and malaria infections respectively, in the region. Similarly, the recovery rate for co-infectious individuals also contributes greatly to reducing the co-infection in the population if its value increases as seen in Figure 5. Another findings obtained is that, increasing dengue vectors contact rate has a great influence on spreading the co-infection in the population. We computed the $R_{0 d m}=19.70>1$, indicating that dengue virus and malaria fever are endemic in the area. Thus, we recommend that center for disease control set out preventive measures in reducing the spread of both diseases and increase the measures on recovery co-infected individuals.

\section{Conclusion and Recommendation}

As demonstrated in this study, the co-infection between dengue virus and malaria fever may have devastating impacts in the tropical/subtropical communities. The model helps in identifying distinct features and underlying relationships between dengue and malaria co-infection. This will be of help to policy makers to devise strategies for controlling the diseases. For future studies, we recommend a formulation with optimal control parameters to determine the strategies for mitigating the spread and control of dengue and malaria co-infection.

Data and materials. The data used for this co-infection model are from previous articles published.

\section{Acknowledgment}

The authors are grateful for the data gathered from the previously published articles. 


\section{References}

[1] P. Yongzhen, L. Shgaoying, L. Shuping, \& L Changguo, "A delay seiqr epidemic model with impulse vaccination and the quarantine measure", Computers and Mathematics with Applications 58 (2009) 135.

[2] W. Viroj, "Concurrent malaria and dengue infection: a brief summary and comment", Asian Pacific Journal of Tropical Biomedicine 1 (2011) 326.

[3] World Health Orginization. Fact sheets/detail/malaria report. 2020.

[4] N. F. B. Simo, J. J. Bigna, S. Kenmoe, S. M. Ndangang, E. Temfack, P. F. Moundipa, \& M. Demanou, "Dengue virus infection in people residing in africa: a systematic review and meta-analysis of prevalence studies", Scientific Reports: Nature Research 9 (2019) 3626.

[5] World Health Organization. Dengue cases report. 2019.

[6] J. A. Mensah, K. I. Dontwi, \& E. Bonyah, "Stability analysis of multiinfections (malaria, zikka dna elephantiasis)", Journal of Advances in Mathematics and Computer Science 30 (2018) 2.

[7] M. J. Mutua, F. B. Wang, \& K N. Vaidya, "Modeling malaria and typhoid fever co-infection dynamics", Journal of Mathematical Bioscience 264 (2015) 128.

[8] A. B. Gumel, Z. Mukandavire, W. Garira, \& J. M. Tchuenche, "Mathematical analysis of a model for hiv-malaria co-infection", Mathematical Biosciences and Engineering 6 (2009) 333. doi:10.3934/mbe.2009.6.333

[9] D. Aldila \& M. Agustin, "A mathematical model of dengue-chikungunya co-infection in aclosed population", Journal of physics: Conf. Series 974 (2018) 012001.

[10] E. Bonyah, M. A. Khan, K. O. Okosun, \& J. F. G. Aguilar, "On the coinfection of dengue fever and zika virus", Optimal and Control App Meth 40 (2018) 394.

[11] O. Olawoyin \& C. Kribs, "Co-infection, altered vector infectivity, and antibody-dependent enhancement: The dengue-zika interplay", Society for mathematical biology 83 (2020) 13.

[12] H. T. Alemmeh, "A co-infection model of dengue and leptospirosis diseases", Advances in Difference Equations 2020 (2020) 664.

[13] T. J. Oluwafemi, E. Azuaba, \& Y. M. Kura, "Stablity analysis of disease free equilibrium of malaria, dengue and typhoid triple infection model", Asian Research Journal of Mathematics, 16 (2020) 11.

[14] T. J. Oluwafemi, N. I. Akinwande, R. O. Olayiwola, \& A. F. Akuta, "Co-infection model formulation to evaluate the transmission dynamics of malaria and dengue fever virus", J. Appl. Sci. Environ. Manage 24 (2020) 7.

[15] O. Gabriel, K. J. Koske, \& M. MutisoJohn, "Transmission dynamics and optimal control of malaria in kenya", Discrete Dynamis in Nature and Society 2016 (2016) 1.

[16] A. Aurelio delos Reyes \& J. M. L. Escaner. "Dengue in the philippines: model and analysis of parameters affecting transmission", Journal of Biological Dynamics 12 (2018) 894.

[17] M. Derouich \& A. Boutayeb, "Dengue fever: Mathematical modelling and computer simulation", Applied Mathematics and Computation 177 (2006) 528.

[18] S. Syafruddin \& N. Lyapunov, "function of sir and seir model for transmission of dengue fever disease", Int. J. Simulation and Process Modelling, 8 (2013) 177.

[19] U. D. P. Fatmawati \& J. Nainggolan, "Parameter estimation and sensitivity analysis of malaria model", Journal of physics Conference Series DOI: 10.1088/1742- 6596/1490/012039 1490 (2020) 012039.

[20] S. Olaniyi \& O. S. Obabiyi, "Mathematical model for malaria transmission dynamics in human and mosquito populations with nonlinear forces of infection", International Journal of Pure and Applied Mathematics 88 (2013) 125.

[21] Y. Xing, Z. Guo, \& J. Liu, "Backward bifurcation in malaria transmission model", Journal of Biological Dynamics 1 (2020) 14.

[22] H. Yin, C. Yang, \& X. Zhang, "Dynamics of malaria transmission model with sterile mosquitoes", J. Bio. Dny. 12 (2018) 577.

[23] C. C. Chavez, Z. Feng, \& W. Huang, "On the computation of $r$ and its role on global stability", //www.researchgate.net/profile/Carlos Castillochavez2/publication/228915276, Biometric Unit Technical Report M1553 (2001) 1. 